\title{
Cobalt Ferrite, a New Gas Sensing Material
}

\author{
Ch. Leroux ${ }^{1}$, M.Bendahan ${ }^{2}$, L.Ajroudi ${ }^{3}$, V.Madigou ${ }^{1}$, N.Mliki $^{3}$ \\ ${ }^{1}$ IM2NP UMR CNRS 6242 Université Sud Toulon Var, BP20132, 83957 La Garde Cedex, France \\ leroux@univ-tIn.fr \\ ${ }^{2}$ IM2NP UMR CNRS 6242, Aix Marseille Université, Av. Escadrille Normandie Niemen, case \\ 152,13397 Marseille Cedex, France \\ 3 LMOP, Faculté des Sciences de Tunis, Université Tunis El Manar, 2090 Tunis, Tunisie
}

\begin{abstract}
:
The electrical response of cobalt ferrites $\mathrm{CoxFe}_{3-\mathrm{x}} \mathrm{O}_{4}$ nanopowders with various cobalt amounts were tested in presence of reducing gases $\left(\mathrm{NH}_{3}\right)$. The morphology, shape and chemical composition of the powders were fully characterized by transmission electron microscopy. For high cobalt amount, the response is typical of a $p$-type semi conductor, as for $x=1$, one observes $n$-type semi conductor response. For $\mathrm{x}=1.8$, fast response and good reversibility was evidenced, with detection even at 10 ppm $\mathrm{NH}_{3}$, well below the accepted level.
\end{abstract}

Key words: cobalt ferrites, nanopowder, $\mathrm{NH}_{3}, \mathrm{CO}$, gas.

\section{Introduction}

In the field of sensors, the last decade was rich in development of ferrite gas sensors in detection of toxic gas and pollution monitoring [1-3]. Measurement and control systems for pollutant and toxic gas emissions gain increasing importance for a sustainable and ecologically responsible development. Sensors based on $\mathrm{CuFe}_{2} \mathrm{O}_{4}$ nanoparticles showed time response in seconds in presence of ethanol gas [4], and sensors based on $\mathrm{MgFe}_{2} \mathrm{O}_{4}$ nanoparticles exhibit selective behavior, depending on the operating temperature [5]. Even the magnetic properties of ferrites were already investigated for a novel magnetic hydrogen sensing [6]. Hence, we were interested in studying cobalt ferrites as nanoparticles or thin films for applications in gas sensors. We concentrated on $\mathrm{Co}_{x} \mathrm{Fe}_{3-\mathrm{x}} \mathrm{O}_{4}$ with $\mathrm{x}=1.8$ as these concentration proved to be the most efficient for catalysis of reducing gases. [7-8]. We have chosen ammonia $\left(\mathrm{NH}_{3}\right)$ gas detection, because it is one of the most important industrial chemicals, used as precursor of various nitrogen compounds (including fertilizers) and as refrigerant gas. As a consequence of its huge toxicity, the acceptable ammonia concentration at the working place is $25 \mathrm{ppm}$ for 8 hours exposure. A relevant ammonia chemical gas sensor must therefore detect ammonia concentrations below this threshold.

\section{Experimental}

Cobalt (II) 2,4-pentanedionate and iron (III) acetylacetonate, were dissolved in benzyl alcohol. The solution was poured into a teflon cup, which was sealed into a steel autoclave, and heated in a furnace at $175{ }^{\circ} \mathrm{C}$ for 48 hours. After cooling, the suspension was washed with ethanol and dichloromethane, sonicated and centrifuged. A black powder was obtained after a final drying at $80{ }^{\circ} \mathrm{C}$. Cobalt ferrites with several concentrations were synthesised, among them $x=1$, and $x=1.8$, which are the two compositions studied in this work. High resolution electron microscopy (HREM) coupled with Energy Dispersive Spectroscopy (EDS) and $X$ rays diffraction were used to characterize the powders at a sub-nanometer scale. $\mathrm{Co}_{x} \mathrm{Fe}_{3}$ $\mathrm{xO}_{4}$ powder were dispersed in terpineol and deposited by solution drop casting on $\mathrm{SiO}_{2} / \mathrm{Si}$ substrates with platinum electrodes. $\mathrm{As} \mathrm{Co}_{x} \mathrm{Fe}_{3}$ $\mathrm{xO}_{4}$ layers are highly resistive, interdigitated electrodes were used in order to reduce the sensor resistance. The distance between the electrodes was $50 \mu \mathrm{m}$. They were obtained from a sputtered $\mathrm{Pt}$ film, using photolithography and lift off processes. The samples were kept in dry air and no conditioning step was carried out before the sensor characterizations. 


\section{Results}

For the first time, successful synthesis of a highly crystalline multi metal oxide by simply dissolving two acetylacetonates in benzyl alcohol was realised [7]. For each cobalt amount, the powder was very homogeneous in composition, size and well dispersed. The size of the particles decreased with the cobalt amount $x$, but was less than $10 \mathrm{~nm}$. With increasing cobalt content, the shape of the particles changed from rounded to a very irregular shape. Fig.1 shows two HREM images of well crystallized cobalt ferrites nanoparticles with different composition, and thus different size and shape.

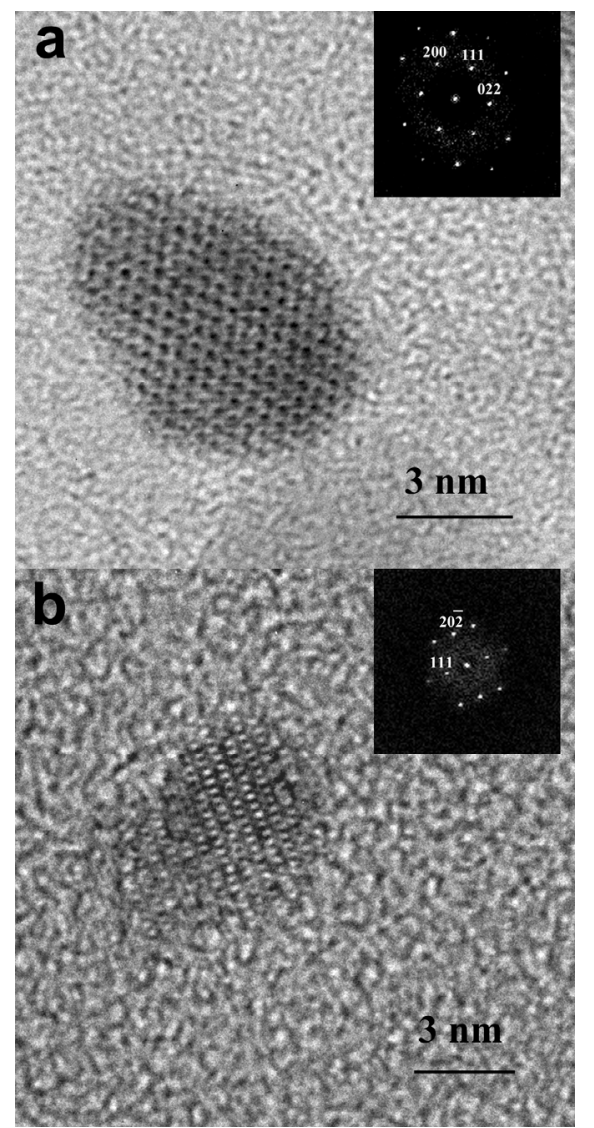

Fig.1.Well crystallized nanoparticles of $\mathrm{Co}_{1.8} \mathrm{Fe}_{2.4} \mathrm{O}_{4}$, with irregular shape, and sizes around $4 \mathrm{~nm}$. The FFT are indexed in the spinel structure.
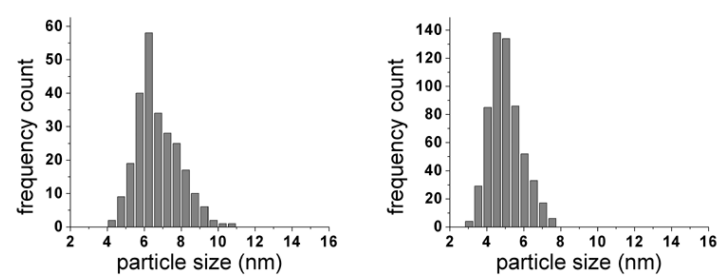

Fig. 2. $\mathrm{CoFe}_{2} \mathrm{O}_{4}$ and $\mathrm{Co}_{1.8} \mathrm{Fe}_{2.4} \mathrm{O}_{4}$ size distribution.
For each composition, narrow log normal size distribution was attained, leading to mean sizes of $6.5 \mathrm{~nm}$ and $4.5 \mathrm{~nm}( \pm 1 \mathrm{~nm}$ ) (Fig.2).

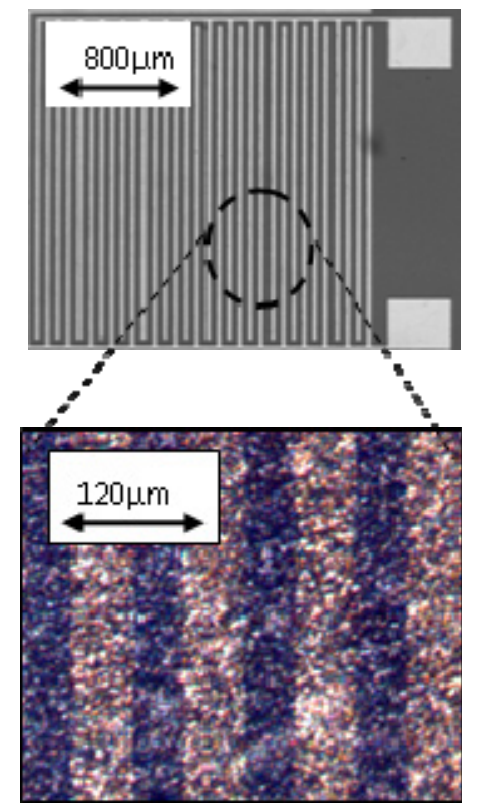

Fig. 3. Optical micrograph of a $\mathrm{Co}_{x} \mathrm{Fe}_{3-x} \mathrm{O}_{4}$ based microsensor

Figure 3 shows the design of the sensor we used to test electrical responses in presence of gas. The optimal working temperature in terms of good sensitivity and fast response was determined. To investigate the $\mathrm{NH}_{3}$ sensing properties of the $\mathrm{Co}_{\mathrm{x}} \mathrm{Fe}_{3-\mathrm{x}} \mathrm{O}_{4}$ films, the sensor devices are introduced in a test chamber allowing the sensor temperature control under variable gas concentrations. Dry synthetic air was used as a reference gas. The sensor performances were tested under diluted gases in synthetic air, at atmospheric pressure. The gas flows were measured through mass flowmeters and the studied concentrations ranged from 10 to $50 \mathrm{ppm}$ with a constant total flow of $0.2 \mathrm{l} / \mathrm{min}$. In order to check the working temperature effect on the sensors response, $\mathrm{Co}_{x} \mathrm{Fe}_{3-\mathrm{x}} \mathrm{O}_{4}$ sensors were maintained at various temperatures. The optimum temperature range, already determined in previous works $[9,10]$, leads us to work between 470-540 K. Figure 4 illustrates the normalized response defined as $R_{\text {gas }} R_{\text {air }}$ to 40 ppm versus working temperature. $R_{\text {gas }}$ is defined as the sensor resistance at various ammonia concentrations, and $R_{\text {air }}$ the sensor resistance in synthetic air. We can notice that the sensor response decreased with increasing operating temperatures above 500 K. This behavior can be explained by considering the surface coverage temperature dependence of the chemisorbed species. 


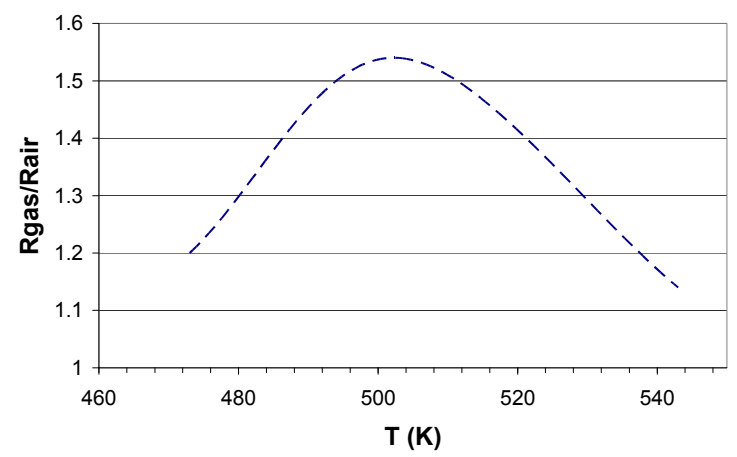

Fig.4. Variation of the sensor normalised response versus temperature. (40 ppm $\mathrm{NH}_{3}$ )

At high temperature, the chemisorptions equilibrium is possible however the coverage decreases with increasing temperature because the desorption rate is greater than the adsorption rate $[11,12]$.Consequently, the sensor was heated at $500 \mathrm{~K}$ and exposed to ammonia concentrations between 10 and 50 ppm (Fig.5). The sensor resistance increased in ammonia presence as expected from the interaction of a reducing gas $\left(\mathrm{NH}_{3}\right)$ with a p-type semiconductor $\left(\mathrm{Co}_{x} \mathrm{Fe}_{3-\mathrm{x}} \mathrm{O}_{4}\right.$ with $\left.\mathrm{x}=1.8\right)$.

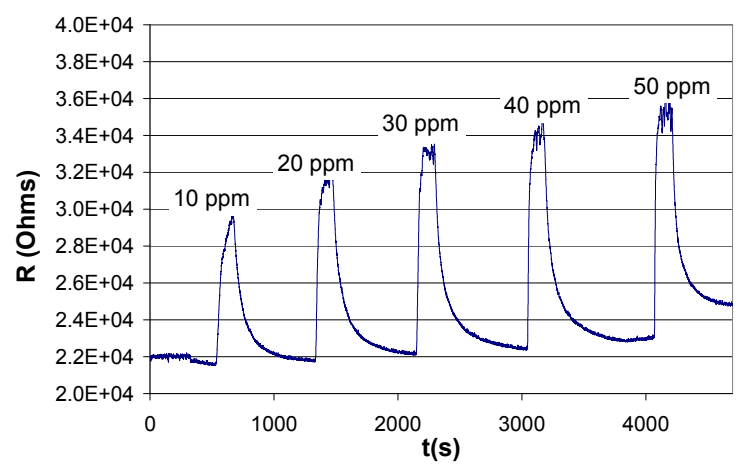

Fig.5. Cobalt ferrite $\mathrm{Co}_{x} \mathrm{Fe}_{3^{-} x} \mathrm{O}_{4} \quad(x=1.8)$ resistance variation with time for various $\mathrm{NH}_{3}$ concentration in dry air, at $500 \mathrm{~K}$

The sensor response at concentrations of 10 and $20 \mathrm{ppm}$ is well pronounced. As the maximum $\mathrm{NH}_{3}$ level authorized at the working place is $25 \mathrm{ppm}$, this result already makes cobalt ferrite an interesting new material for $\mathrm{NH}_{3}$ sensing. Preliminary results on $\mathrm{CoFe}_{2} \mathrm{O}_{4}$ showed that the resistance of this compound decreases in presence of a reducing gas (CO).

\section{Acknowledgements}

Authors acknowledge the PHC-Utique French Tunisian exchange program $11 \mathrm{G} 1301$ for financial support.

\section{References}

[1] A. Virden, S. Wells, K. O'Grady J. Magn. Magn. Mater., 316, 768-771 (2007), doi:10.1016/j.jmmm.2007.03.100

[2] D.S. Mathew, R.S. Juang, Chem. Eng. J.,129, 51-65 (2007). doi:10.1016/j.cej.2006.11.001

[3] M. Sugimoto J. Am. Ceram. Soc., 82, 269 (1999).

[4] Z.Sun, L. Liu, D. Z. Jia, W.Pan, Sensors and actuators $B, \quad 125, \quad 144-148, \quad$ (2007), doi:10.1016/j.snb.2007.01.050

[5] Y.-L. Liu, Z.-M. Liu, Y. Yang, H.-F. Yang, G.-L. Shen, R.-Q. Yu, Sensors and Actuators B, 107, 600-604, (2005) doi:10.1016/j.snb.2004.11.026

[6] A.Punnoose, K. M. Reddy, A. Thurber, J.Hays, M. H. Engelhard, Nanotechnology, 18, 165502, (2007), doi:10.1088/0957-4484/18/16/165502

[7] L. Ajroudi, V. Madigou, S. Villain, N. Mliki, Ch. Leroux Journal of Crystal Growth 312, 24652471 (2010) doi:10.1016/j.jcrysgro.2010.05.024

[8] L. Ajroudi, V.Madigou, S.Villain, N.Mliki, Ch.Leroux Sensor Letters, 9, 1-4 (2011), doi: 10.1166/sl.2011.1798

[9] M. Bendahan, R. Boulmani, J.L. Seguin and K. Aguir, Sensors and Actuators B: Chemical, 100 (2004), p. 320-324. doi:10.1016/j.snb.2004.01.03

[10] J. Guérin, M. Bendahan, K. Aguir, Sensors and Actuators B: Chemical, 128 (2008) p. 462-467. http://dx.doi.org/10.1016/j.snb.2007.07.010,

[11] M. Bendahan , J. Guérin, R. Boulmani, K. Aguir, Sensors and Actuators B: Chemical, 124 (2007), p. 24-29.; doi:10.1016/j.snb.2006.11.036

[12] J. Guérin, K. Aguir, M. Bendahan, C. LambertMauriat, Sensors and Actuators B: Chemical, 104 (2005), p. 289-293; doi:10.1016/j.snb.2004.05.019 